\title{
REVIEW
}

\section{NETosis contributes to the pathogenesis of diabetes and its complications}

\author{
Rachel Njeim ${ }^{1,2, *}$, William S Azar(D1,2,3,*, Angie H Fares1,2, Sami T Azar2,4, Hala Kfoury Kassouf ${ }^{5}$ and Assaad A Eid1,2 \\ 'Department of Anatomy, Cell Biology and Physiological Sciences, Faculty of Medicine and Medical Center, American University of Beirut, Beirut, Lebanon \\ 2AUB Diabetes, American University of Beirut, Beirut, Lebanon \\ ${ }^{3}$ Department of Physiology and Biophysics, Georgetown University Medical Center, Washington, District of Colombia, USA \\ ${ }^{4}$ Department of Internal Medicine, Faculty of Medicine and Medical Center, American University of Beirut, Beirut, Lebanon \\ ${ }^{5}$ Department of Pathology and Laboratory Medicine, Faculty of Medicine and Medical Center, American University of Beirut, Beirut, Lebanon
}

Correspondence should be addressed to H Kfoury Kassouf or A A Eid: hk113@aub.edu.lb or ae49@aub.edu.Ib

*(R Njeim and W S Azar contributed equally to this work)

\begin{abstract}
NETosis, a novel form of neutrophil-related cell death, acts as a major regulator of diabetes and diabetes-associated complications. In this review, we show that the extrusion of neutrophil extracellular traps, termed NETs, plays an important role in the pathogenesis of type 1 diabetes mellitus (T1DM), type 2 diabetes mellitus (T2DM), and diabetes-induced complications. In T1DM, $\beta$-cell death induces the sequestration of neutrophils in the pancreas and seems to be correlated with increased NETosis. In T2DM patients, products of NETs release are significantly elevated. Increased levels of dsDNA are correlated with the presence of cardiovascular disease and diabetic kidney disease, further supporting the role of NETosis in the pathogenesis of other diabetesinduced complications such as impaired wound healing and diabetic retinopathy. NETosis is induced by high glucose through incompletely understood mechanisms, but it also appears to be elevated in patients with diabetes who have tightly controlled glucose levels. We hypothesize that hyperglycemia worsens the already elevated baseline of NETosis in diabetic patients to further increase its detrimental effects.
\end{abstract}

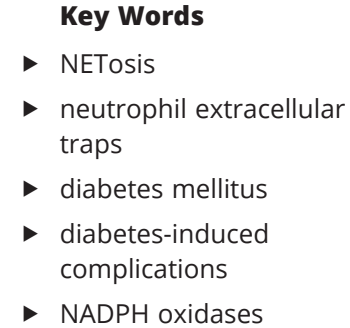

Journal of Molecular Endocrinology (2020) 65, R65-R76

\section{Introduction}

Polymorphonuclear neutrophils (PMNs), the most abundant leukocytes in the blood, are considered part of the first-line immune defense and play a central role during the innate immune response to protect the body against invading pathogens, such as bacteria, fungi, protozoa, and viruses (Rosales et al. 2017). Upon infection or inflammation, PMNs are the first leukocytes to migrate from the blood to injured or infected sites where they phagocytose invading microorganisms in order to eliminate them via different cytotoxic mechanisms (Rosales et al. 2017). Neutrophils target these invading microorganisms through three different mechanisms: (1) phagocytosis and degradation through the synthesis of reactive oxygen species (ROS) in the phagolysosomes, (2) degranulation or the release of antimicrobial peptides, and, (3) as recently described, microbial trapping through the release of extracellular DNA traps termed neutrophil extracellular traps (NETs) (Papayannopoulos 2018).

NETosis was first described in 1996 by Takei et al. as a cell death pathway that differs from necrosis and apoptosis (Takei et al. 1996). Phorbol 12-myristate 13-acetate (PMA), a potent activator of neutrophils, is used to elucidate 
the relationship between activation of neutrophils and their subsequent death. Clear morphological changes are observed in PMA-treated neutrophils, starting with a fusion of the multilobulated nucleus, followed by reduced chromatin condensation, and soon after, by a disruption of the nuclear envelope with cytoplasmic organelles remaining intact. This mechanism is described to be dependent on reactive oxygen species production (Takei et al. 1996). In 2004, Brinkmann et al. further interpreted this unusual cell death process and termed it NETosis (Brinkmann et al. 2004).

NETosis is an essential process of the innate immune response. It is triggered by both infectious and 'sterile' stimuli such as cytokines, cholesterol crystals, monosodium uric acid crystals, immune complexes, and autoantibodies (Keshari et al. 2012, Schorn et al. 2012, Behnen et al. 2014, Yalavarthi et al. 2015). Moreover, NETosis is shown to play a role in the adaptive immune response where neutrophils engage with T cells, B cells, and antigen-presenting cells (Mantovani et al. 2011). Also, NETosis is shown to stimulate the immune system during an inflammatory response (Garcia-Romo et al. 2011, Semeraro et al. 2011).

This process of NETosis leads to the release of neutrophil extracellular traps (NETs), which are large, web-like structures composed of decondensed chromatin associated with granules of degradative enzymes (Brinkmann et al. 2004). NETs prevent the migration of extracellular pathogens, and neutralize and kill bacteria, fungi, viruses and parasites (Papayannopoulos 2018). NETs are ultimately cleared either through the engulfment by macrophages through an active endocytic process or by DNAse I, an endonuclease that cleaves chromatin within NETs (Hakkim et al. 2010, Farrera \& Fadeel 2013). Despite a beneficial effect of NETs production in pathogens clearance, there is increasing evidence that excessive NETs formation may contribute to the pathogenesis of autoimmune diseases including rheumatoid arthritis (Corsiero et al. 2016), systemic lupus erythematosus (Cooper et al. 1999), vasculitis, and, recently, diabetes (Wong et al. 2015, Corsiero et al. 2016, Qin et al. 2016). Herein, we describe and discuss the underlying mechanisms of NETosis and its role in the pathogenesis of diabetes and diabetes-induced microvascular complications.

\section{NETosis a neutrophil-specific cell death process}

NETs are produced through a cell death process termed NETosis. To date, three models of NETosis have been described: suicidal NETosis, vital NETosis with the release of nuclear DNA, and vital NETosis with release of mitochondrial DNA (Delgado-Rizo et al. 2017).

\section{Conventional suicidal NETosis}

Suicidal NETosis, which lasts 2-4 h (Pilsczek et al. 2010), remains the most elucidated model. In this model, neutrophils are first activated by ligand binding to neutrophil toll-like receptors and receptors for IgG-Fc, complement, or cytokines (Yang et al. 2016). Upon activation, the endoplasmic reticulum releases its stored calcium ions into the cytoplasm (Yang et al. 2016). This increase in cytosolic calcium cations then activates the protein kinase $\mathrm{C}$ (PKC)/Raf/MEK/ERK pathway, leading to gp91phox phosphorylation for the activation of NADPH oxidase (NOX) complex and the subsequent generation of reactive oxygen species. Cytosolic $\mathrm{Ca}^{2+}$ cations also act as cofactors for peptidyl arginase deaminase 4 (PAD4), an enzyme that catalyzes the hydrolysis of peptidyl-arginine to form peptidyl-citrulline on histones. This process, termed histone citrullination, promotes the loss of positive charges required for the interaction of histones with DNA, and, hence, leads to the decondensation of chromatin ( $\mathrm{Li}$ et al. 2010, Lewis et al. 2015). In suicidal NETosis, reactive oxygen species behave as second messengers promoting neutrophil elastase (NE) and myeloperoxidase (MPO) translocation from azurophilic granules to nucleus. NE proteolytically processes histones to disrupt chromatin packaging, while MPO binds chromatin and synergistically with NE, promotes chromatin decondensation (Papayannopoulos et al. 2010). This enhances the gradual separation and loss of the nuclear membrane, which disintegrates into smaller individual vesicles. Decondensed chromatin then associates with cytoplasmic proteins and granule toxins. Finally, the DNA is released as extracellular traps (ETs) secondary to cellular lysis, ultimately entrapping exogenous bacteria (Brinkmann \& Zychlinsky 2007, Neeli et al. 2008, 2009, Li et al. 2010, Lewis et al. 2015). Conventional suicidal NETosis differs from programmed cell death or apoptosis. In the event of apoptosis, DNA condenses first then fragments (Hamam \& Palaniyar 2019b). It is suggested that post-translational modifications (PTMs) of histones including acetylation appear to play a central role in regulating neutrophil death (Hamam \& Palaniyar 2019b). Histone acetylation was recently found to promote NETosis at baseline when induced by NOX-dependent or NOX-independent pathway agonists in human neutrophils (Hamam et al. 2019). In general, histone acetylation results in chromatin 
decondensation. However, increased levels of histone acetylation was shown to switch neutrophil death from NETosis to apoptosis by activating the cleavage of caspase-3 (Hamam \& Palaniyar 2019a). Interestingly, in the presence of NETosis-promoting stimuli, high levels of histone deacetylase inhibitors (HDACis) inhibit both NETosis and apoptosis. Taken together, both histone citrullination and acetylation of histones have been found to alter NETosis and apoptosis with the net outcome depending upon the degree of PTMs of histones and the presence or absence of NETosis-inducing stimuli (Hamam \& Palaniyar 2019b).

\section{Vital NETosis}

In vital NETosis, also termed nonlytic NETosis, neutrophils release NETs without losing their plasma membrane. It happens within 5-60 min in response to Staphylococcus aureus (Pilsczek et al. 2010) and Candida albicans (Byrd et al. 2013) and does not require the generation of reactive oxygen species (Delgado-Rizo et al. 2017). The sequential steps of vital NETosis occur as follows: (1) recognition of the stimuli through toll-like receptors (TLRs) and the complement receptor for C3 protein (Clark et al. 2007, Byrd et al. 2013); (2) loss of the lobular and multinucleated shape of the nucleus; (3) nuclear envelope disruption and budding of vesicles; (4) vesicles containing DNA filaments approach the intact plasma membrane; (5) release of DNA as ETs through a small area in cell surface and release of some cytoplasmic granules into the extracellular space to associate with DNA (Delgado-Rizo et al. 2017). In vital NETosis, the release of nuclear content does not affect the lifespan of the neutrophils. In fact, these neutrophils retain their ability to phagocytose pathogens (Yipp \& Kubes 2013).

Additionally, a second type of vital NETosis was described. It involves the release of mitochondrial DNA instead of nuclear DNA and is dependent on ROS production. The use of diphenyleneiodonium (DPI), an inhibitor of NADPH oxidases, resulted in a complete block of mitochondrial DNA release from neutrophils, suggesting that this type of NETosis is also dependent on NADPH oxidases. This process results in NETs formation within $15 \mathrm{~min}$ through the recognition of $\mathrm{C5a}$ or lipopolysaccharide (LPS) (Yousefi et al. 2009).

\section{Diabetes mellitus and the widespread consensus on tight glycemic control}

While NETosis was first described not much more than two decades ago, research on diabetes mellitus (DM) was initiated about a century ago. DM is defined as a group of metabolic diseases clinically characterized by hyperglycemia that results from defects in insulin action, insulin secretion, or both. Despite the different etiologies of type 1 (insulin deficiency) and type 2 diabetes (insulin resistance), both are associated with 'macrovascular complications', including cardiovascular disorders, heart failure, atherosclerosis, and cerebrovascular events, as well as with 'microvascular' complications, such as diabetic neuropathy, diabetic retinopathy, and diabetic kidney disease (Forbes \& Cooper 2013). Herein, we will be focusing on the diabetes-induced microvascular complications.

Achieving optimal glycemic control as early as possible in the course of the disease has always been the ultimate goal of diabetes management. In type 1 diabetic subjects, rapid-acting and long-acting insulin analogues improve glycemic control. In type 2 diabetes, there are a range of pharmacological agents used to attain normoglycemia including insulin-sensitizing agents such as metformin and thiazolidinediones, drugs that stimulate the release of insulin from the pancreas, namely sulfonylureas, glucagon-like peptide-1 (GLP-1) agonists, and dipeptidyl peptidase-IV (DPPIV) inhibitors, as well as sodiumglucose transport protein 2 (SGLT2) inhibitors, which prevent reabsorption of glucose in the kidneys (Forbes \& Cooper 2013).

Randomized controlled trials (RCTs) emphasize the significance of tight glycemic control in preventing the onset or progression of diabetes-associated complications. The Diabetes Control and Complications Trial (DCCT) and Epidemiology of Diabetes Interventions and Complications (EDIC) trial in type 1 diabetes have shown that tight glycemic control ameliorated but did not halt the progression of the microvascular diabetic complications (Skyler 2004, Nathan \& DCCT/EDIC Research Group 2014). In type 2 diabetes, the RCTs were equivocal. While the UK Prospective Diabetes Study (UKPDS) showed a significant risk reduction in diabetic kidney disease and diabetic retinopathy after 10 years of intensive glycemic control (King et al. 1999), other RCTs questioned the implication of tight glycemic control in all diabetic patients. For example, The Action to Control Cardiovascular Risk in Diabetes (ACCORD), Action in Diabetes and Vascular Disease (ADVANCE) and Veteran Affairs Diabetes Trial (VADT) all showed that tight glycemic control does not reduce macrovascular disease in older patients with diabetes who are at risk for or have cardiovascular disease, and improvement in microvascular complications were relatively modest and 
limited to reduced proteinuria (Terry et al. 2012). In fact, intensive glycemic control was associated with increased mortality in the ACCORD study, and with more frequent and severe episodes of hypoglycemia in the ACCORD and VADT trials (Terry et al. 2012). Collectively, these RCTs have shown that it is important to reevaluate the wellknown consensus on tight glycemic control to prevent diabetes-associated microvascular and macrovascular complications. More importantly, these trials highlight that glucose alone is not accountable for the pathogenic manifestations seen in diabetes mellitus, paving the way for identifying adjunct therapy to halt the development or progression of diabetes-induced complications, or even reverse their outcomes.

\section{The role of NETosis in the pathogenesis of type 1 diabetes mellitus}

Neutrophils and NETs were recently shown to be involved in the pathogenesis of type 1 diabetes mellitus (Table 1). The inhibition of neutrophil function and NETs formation attenuates the development of type 1 diabetes (Diana et al. 2013). In experimental models of type 1 diabetes, physiological $\beta$-cell death induced the recruitment and activation of $\mathrm{B}-1 \mathrm{a}$ cells, neutrophils and plasmacytoid dendritic cells (pDCs) to the pancreas. Subsequently, dsDNA-specific IgGs were secreted from activated B-1a cells, stimulating neutrophils to release DNA-binding cathelicidin-related antimicrobial peptide (CRAMP), which binds self-DNA. Self-DNA, dsDNAspecific IgGs and CRAMP peptide then activated pDCs leading to interferon- $\alpha$ production. Also, histological analysis confirmed the presence of NETs associated with CRAMP release (Diana et al. 2013). In that same spirit, a study conducted on 107 adult patients with newly diagnosed type 1 diabetes and 21 children with persistent islet autoantibodies and a family history of type 1 diabetes revealed a significantly lower neutrophil count compared to age and gender-matched control subjects. Notably, all subjects had no laboratory signs of acute infection (Harsunen et al. 2013). Further studies aiming to understand the mechanism behind the perturbation of neutrophil homeostasis in type 1 diabetes have shown that the circulating neutropenia was not caused by impaired neutrophil output from the bone marrow or by impaired differentiation, but rather by an increased sequestration of the neutrophils in the pancreatic tissue (Valle et al. 2013). In an effort to correlate NETosis with $\beta$-cells death in type 1 diabetes, it was reported that the circulating protein levels and enzymatic activities of proteinase 3 (PR3) and

Table 1 Neutrophil extracellular traps (NETs) in patients with type 1 diabetes mellitus.

\begin{tabular}{l} 
Study reference \\
\hline Harsunen et al. \\
2013
\end{tabular}

Valle et al. 2013

Wang et al. 2014

Subjects

- 107 adult patients with newly diagnosed T1DM

- 21 children with persistent islet autoantibodies and a family history of T1DM

- 1238 age and gender matched control subjects

- Pediatric patients with T1DM at onset

- Healthy pediatric controls

- Adult patients with T1DM at onset

- Adult patients with long-standing T1DM and T2DM

- Healthy adult controls

- Relatives of patients with T1DM

- A total of 149 children with T1DM and 77 age- and sex-matched healthy control subjects

Wong et al. 2015 - Neutrophils isolated from patients with T1DM

Qin et al. 2016 - Forty-four subjects with T1DM with a median age of 26.5 years and median disease duration of $2 \cdot 2$ years and 38 age-matched controls
Findings pertinent to NETosis

- Decreased neutrophil count in adult patients with newly diagnosed T1DM and in children with persistent islet autoantibodies

- Peripheral neutropenia precedes and accompanies the onset of T1DM

- Neutrophils were sequestered in the exocrine pancreas of multiorgan donors with T1DM (at onset and at later stages of the disease)

- Circulating protein levels and enzymatic activities of PR3 and NE were significantly elevated in patients with T1DM

- Circulating NE and PR3 levels were positively correlated with autoantibodies against $\beta$-cell antigens

- A marked increase in circulating MPO-DNA complexes was observed in patients with T1DM

- Expression of PAD4 was elevated in neutrophils from individuals with diabetes

- NE and PR3 were significantly reduced in patients with T1DM, particularly in subjects within 3 years of diagnosis.

- A decrease in neutrophil counts in recent-onset T1DM was observed

- NE and PR3 levels were correlated with absolute neutrophil counts 
neutrophil elastase (NE) were significantly elevated in patients with type 1 diabetes, especially in patients with disease duration of less than 1 year, compared to age and gender-matched control subjects. The increase in NE and PR3 was positively correlated with the increase of the positive numbers and titers of the autoantibodies against $\beta$-cell antigens. However, it was noted that an elevation was also observed in autoantibody-negative patients. In line with the increased NE and PR3 levels, a marked increase of circulating MPO-DNA complexes was seen in patients with type 1 diabetes as compared to healthy subjects. Taken together, these observations suggest that the increase in circulating NE and PR3 protein levels may be associated with enhanced neutrophil extracellular traps formation (Wang et al. 2014). To further investigate the link between $\mathrm{NE}, \mathrm{PR} 3$, and the onset of type 1 diabetes, changes in the expression of NE and PR3 were determined in non-obese diabetic (NOD) mice from 2 to 30 weeks of age. It was shown that the enzymatic activities of circulating NE and PR3 were significantly elevated and sustained before the onset of diabetes. Intriguingly, the NE/PR3 activities in diabetic mice gradually decreased to baseline levels, most likely due to the termination of autoimmune response after complete $\beta$-cell destruction (Wang et al. 2014).
These findings support the role of NETosis in the pathogenesis of $\beta$-cell autoimmunity and highlight NE and PR3 as sensitive biomarkers for the diagnosis of type 1 diabetes. Nevertheless, in a recent study, it was stated that both NE and PR3 were significantly reduced in patients with type 1 diabetes, particularly in subjects within 3 years of diagnosis. This is associated with the known decrease in neutrophil counts in recent-onset type 1 diabetes (Qin et al. 2016). Therefore, circulating levels of PR3 and NE generally reflect neutrophil counts. Even if found to be low, they should not rule out the presence of NETosis in type 1 diabetes.

\section{The role of NETosis in the pathogenesis of type 2 diabetes mellitus}

In line with the results obtained in type 1 diabetes, increasing evidence has shed light on the role of neutrophils and NETosis in the pathophysiology of type 2 diabetes (Table 2). Considering that NETosis occurs during inflammation and that patients with type 2 diabetes are characterized by a state of low-grade chronic inflammation, NETosis was evaluated in the sera of 38 patients with type 2 diabetes and 38 age and gender-matched non-diabetic

Table 2 Neutrophil extracellular traps (NETs) in patients with type 2 diabetes mellitus.

\begin{tabular}{|c|c|}
\hline Study reference & Subjects \\
\hline Joshi et al. 2013 & $\begin{array}{l}\text { - } \quad \text { Patients with T2DM }(n=18) \\
\text { - } \quad \text { Healthy donors }(n=17)\end{array}$ \\
\hline $\begin{array}{l}\text { Menegazzo et al. } \\
2015\end{array}$ & $\begin{array}{l}\text { - } 38 \text { T2D and } 38 \text { age- and sex-matched } \\
\text { non-diabetic individuals }\end{array}$ \\
\hline
\end{tabular}

Wong et al. 2015 - Neutrophils isolated from patients with T2DM

Carestia et al. 2016 - Recently diagnosed T2DM patients and after 6 and 12 months of metformin treatment (500-2500 mg/daily) $(n=25)$

- Healthy Donors $(n=25)$

Menegazzo et al. 2018

\section{Findings pertinent to NETosis}

- Neutrophils from diabetic subjects make inadequate and inactive NETs.

- T2D patients showed increased neutrophil elastase, mono- and oligonucleosomes, and cell-free dsDNA as compared to the control subjects

- A positive correlation was observed between $\mathrm{HbA1c}$ and mono- and oligonucleosomes

- dsDNA was positively correlated with the presence of nephropathy and cardiovascular disease

- Serum IL-6 concentrations were higher in T2D patients as compared to control subject and correlated with serum dsDNA levels

- Expression of PAD4 was elevated in neutrophils from individuals with diabetes

- Basal levels of NETs in patients with recently diagnosed T2DM patients were higher compared to healthy donors.

- Neutrophils from T2D patients did not respond to further stimulation by PMA or TNF $\alpha$

- NET formation, nucleosomes, and NE-dsDNA complexes were still elevated in the plasma of patients with T2D after 6 months of treatment.

- Neutrophil functional responses were restored to normal after 12 months of treatment

- Metformin significantly decreased the concentrations of neutrophil elastase, PR3, histones and dsDNA, whereas insulin or dapagliflozin had no significant effect. 
control subjects (Menegazzo et al. 2015). Type 2 diabetic patients showed increased NETosis products, including neutrophil elastase, mono- and oligonucleosomes, and cell-free double stranded DNA (dsDNA), as compared to the control subjects. More importantly, a positive correlation was observed between HbA1c and mono- and oligonucleosomes, while dsDNA was correlated with the presence of nephropathy and cardiovascular disease. Also, serum concentration of interleukin-6 (IL-6) and tumor necrosis factor alpha (TNF $\alpha$ ), markers of systemic low-grade inflammation, which are also known to be NET inducers, were shown to be elevated in patients with type 2 diabetes as compared to the control subjects, and to be directly correlated with dsDNA concentrations (Menegazzo et al. 2015). It was also reported that high glucose ( $25 \mathrm{mM})$ significantly increased the rate of NETosis and release of NETs in vitro, with and without PMA, as compared to 5 $\mathrm{mM}$ glucose and $25 \mathrm{mM}$ mannitol (Menegazzo et al. 2015). To assess the effect of metabolic control, NETosis was re-evaluated in diabetic patients after metformin treatment (500-2500 mg/daily) (Carestia et al. 2016). Despite the restoration of glucose and $\mathrm{HbA1c}$ levels to normal levels after 6 months of metformin treatment, NET formation, nucleosomes, and NE-dsDNA complexes were still present in the plasma of diabetic patients. However, these neutrophil functional responses were restored to normal levels after 12 months of treatment with no change in neutrophil count across this period. Indeed, neutrophils from diabetic patients at time of diagnosis had elevated levels of basal NET production compared to control subjects. Interestingly, neutrophils from diabetic patients did not respond to further stimulation by PMA or tumor necrosis factor alpha (TNF $\alpha)$ as did the neutrophils of healthy donors. This supports the fact that neutrophils from diabetic patients already possess increased basal NET production and are unable to further undergo NETosis (Carestia et al. 2016). This study shows, for the first time, that despite the normalization of glucose levels, NETosis remained elevated, suggesting that increased NET formation is not a consequence of impaired glycemic control. To further assess the effect of metformin on NETosis, NET products were assessed in the plasma of patients with pre-diabetes who were randomly assigned to receive either metformin or placebo for 2 months. Additionally, in order to control for the effect of glucose, NET components were also evaluated in the plasma of patients with type 2 diabetes before and after treatment with dapagliflozin or insulin (Menegazzo et al. 2018). After 2 months of treatment, patients in the metformin group showed a significant decrease in the concentrations of dsDNA, neutrophil elastase, proteinase-3, and histones as compared to patients in the placebo group. Notably, no changes were observed in glucose levels or insulin sensitivity. Moreover, in patients who initiated basal insulin therapy or dapagliflozin, circulating biomarkers were not significantly reduced after 3 months of treatment as compared to baseline. These observations suggest that the observed effects of metformin on NETosis were independent of glucose control. In vitro, metformin significantly blunted NETosis in PMA and ionomycinstimulated neutrophils. Also, metformin prevented membrane translocation of protein kinase C-beta II isoform (PKC- $\beta$ II), which is a mediator of hyperglycemiainduced chronic complications, and activation of NADPH oxidase in neutrophils, which is required for neutrophil oxidative burst and is a critical step of NOX-dependent NETosis, both of which decreased NETosis response (Menegazzo et al. 2018). These findings highlight that the inhibition of NETosis is a new promising pharmacological effect that can be exerted by metformin. More importantly, expression of peptidylarginine deiminase 4 (PAD4), a crucial enzyme in chromatin decondensation and in mediating NETosis, was shown to be significantly elevated in patients with type 1 or type 2 diabetes as compared to healthy controls (Wong et al. 2015). Additionally, basal calcium levels were markedly elevated in patients with type 2 diabetes, and intracellular calcium levels were found to be positively correlated with fasting serum glucose levels (Alexiewicz et al. 1995), reminding us of the role of calcium in NET formation as it induces the generation of reactive oxygen species and PAD4-mediated histone citrullination and subsequent chromatin decondensation. Furthermore, NETosis was shown to metabolically require glucose (Rodriguez-Espinosa et al. 2015), therefore, elevated glucose is associated with NETosis at many levels. In contrast to these observations, Joshi et al. have previously shown that high glucose impairs and delays NETs formation as T2DM neutrophils possessed elevated basal NET formation and are unable to undergo further NETosis in response to external stimuli (Joshi et al. 2013). These differences may be attributed to the nature of the stimuli used in each study.

\section{The role of NETosis in the pathogenesis of diabetes-induced microvascular complications}

In addition to its potential role in the development of type 1 and type 2 diabetes, NETosis is also thought to be involved in promoting diabetes-induced complications (Fig. 1) 
(Table 3). Indeed, NETs are implicated in diabetic wound healing, frequently associated to diabetic patients with distal symmetric polyneuropathy (DSP) (Forbes \& Cooper 2013, Callaghan et al. 2015, Wong et al. 2015, Fadini et al. 2016, Volmer-Thole \& Lobmann 2016, Das et al. 2018). When exposed to excisional skin wounds, diabetic mice had higher levels of citrullinated histone H3 (H3Cit), a marker for NETosis, in their wounds, and healing was delayed when compared to WT normoglycemic mice. DNase I, an enzyme involved in the disruption of NETs, accelerated wound healing in diabetic and WT mice. To further establish the role of NETosis in wound healing, protein arginine deiminase knockout (Padi4 ${ }^{-/-}$) mice were used.
In contrast to the robust increase of H3Cit signals that were detected in the WT wounds, no H3Cit was observed in wounds from Padi4-/- mice, despite normal neutrophil recruitment. Notably, Padi4-/- mice did not possess clinical signs of infection and their wound healing was accelerated. This may be explained by the fact that, in Padi4-/- mice, in addition to a decrease in NETosis, other neutrophil functions such as phagocytosis remained intact. Intriguingly, accelerated wound healing was not compromised by inducing type 1 diabetes in Padi4-/mice, suggesting that diabetes delays wound healing by activating NETosis. PAD4 inhibition and DNase 1 may thus serve as potential therapeutic approaches to

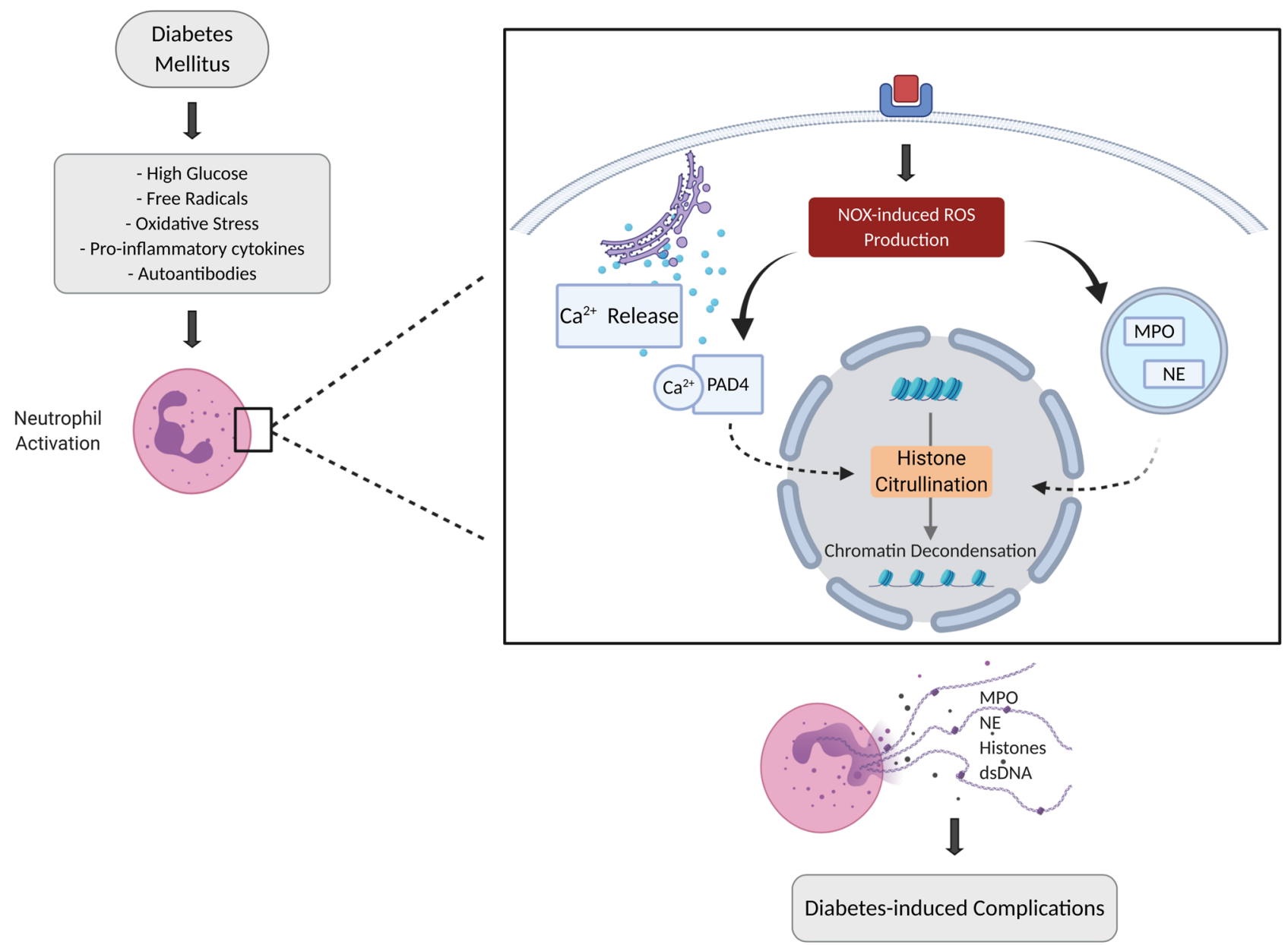

\section{Figure 1}

Schematic diagram representing the key role of NETosis in the development and/or progression of diabetes-induced complications. Hyperglycemia increases the production of free radicals resulting in oxidative stress. It is also associated with elevated levels of both proinflammatory cytokines and autoantibodies. This, in turn, promotes neutrophil recruitment and activation. An increase in cytosolic calcium cations activates NADPH oxidases and acts as a cofactor for PAD4. PAD4 catalyzes histone citrullination inducing chromatin decondensation. Increased reactive oxygen species behave as second messengers promoting NE and MPO translocation to the nucleus and enhancing chromatin decondensation. The DNA is then released with toxic granules and cytoplasmic proteins as extracellular traps secondary to cellular lysis, eventually leading to diabetes-associated complications. PAD4, peptidylarginine deiminase 4; MPO, myeloperoxidase; NE, neutrophil elastase. A full-colour version of this figure is available at https://doi.org/10.1530/ JME-20-0128. 
Table 3 Neutrophil extracellular traps (NETs) in patients with diabetes-induced complications.

Study
reference

Fadini et al. 2016

\section{Subjects}

- Control subjects without diabetes

- Patients with diabetes

- Patients with DFUs
Yang et al. 2019

Park et al. 2016

Wang et al. 2018

Miyoshi et al. 2016
- 14 diabetic foot patients (seven males and seven females)

- 14 healthy volunteers (seven males and seven females)

- Of 88 patients with T2DM, 26 patients with diabetic retinopathy and 62 patients without diabetic retinopathy

- 28 healthy controls

- Healthy controls $(n=27)$

- Patients with T2DM $(n=90)$

- Seventy-five T2DM patients were divided into three groups: diabetes without retinopathy (DWR) $(n=30)$, non-proliferative diabetic retinopathy (NPDR) $(n=29)$, and proliferative diabetic retinopathy (PDR) $(n=31)$

- 11 well-controlled patients with T2DM patients

- 13 healthy volunteers

\section{Findings pertinent to NETosis}

- NET components were increased in nonhealing human DFUs

- Increased concentrations of NE in the wound was correlated with infection and worsening of the ulcer

- Elastase, histones, neutrophil gelatinase-associated lipocalin, and PR3 levels were increased in the blood of patients with DFUs

- Circulating elastase and PR3 were associated with infection

- Serum elastase predicted delayed wound healing

- Neutrophils isolated from patients with DFU possessed increased spontaneous NETosis but impaired inducible NETosis

- dsDNA and NE were significantly elevated in diabetic foot patients

- Circulating DNA-histone complex and polymorphonuclear NE levels were significantly increased in patients with diabetic retinopathy compared with those without retinopathy

- DNA-histone complex and NE were independent risk factors of retinopathy

- NETs formation was increased in the sera of patients with diabetic retinopathy irrespective of the disease stage

- Serum MPO-DNA complex levels were significantly increased in well-controlled patients with T2DM and positively correlated with clinical/laboratory parameters which are known to be risk markers for diabetic nephropathy or diabetic kidney disease wound healing (Wong et al. 2015). PAD4 inhibition with Cl-amidine reduced NETs formation and rescued wound healing in diabetic mice (Fadini et al. 2016), and the inhibition of PKC $\beta$ II also prevented exaggerated NET production in diabetic mice and promoted accelerated wound healing (Das et al. 2018). Of interest, PKC $\beta I I$ is tightly correlated to phosphorylation of the p47phox thus activating Nox 2 and leading to ROS production (Fontayne et al. 2002). To validate these pre-clinical findings, the effect of NETosis on delayed wound healing was established in patients with diabetes. Using proteomics, NET products were found to be elevated in nonhealing human diabetic foot ulcers (DFUs). Moreover, since DFUs are associated with systemic inflammation, NETosis biomarkers were assessed in the bloodstream of diabetic patients with DFUs compared with matched diabetic patients without DFUs. NET components, including elastase, histones, neutrophil gelatinase-associated lipocalin, and proteinase-3, were found to be elevated in blood of patients with diabetic foot ulcers. Additionally, a high concentration of circulating elastase and proteinase-3 was shown to be associated with infection, while serum elastase predicted delayed healing (Fadini et al. 2016). Cumulatively, these data imply that NETosis delays DFU healing. Consistent with these findings, a recent study revealed high levels of NET products in the sera of diabetic foot patients (Yang et al. 2019). Also, wound healing was significantly delayed in diabetic mice lacking leptin receptor $\left(L e p R^{\mathrm{db} / \mathrm{db}}\right)$, accompanied with high NETs levels, as compared to normoglycemic control mice $\left(L e p R^{\mathrm{m}+/ \mathrm{db}}\right)$. Furthermore, dsDNA, PAD4 and citrullinated histone $\mathrm{H} 3$ were all significantly elevated in an in vitro PMAinduced NETosis model. Importantly, for the first time, the study demonstrated that treatment with hydrogen sulfide (H2S), an endogenous signaling molecule with antioxidant effects, significantly inhibited NETosis and 
lead to accelerated wound healing in $L e p R^{\mathrm{db} / \mathrm{db}}$ mice. The inhibitory effect of H2S on NETs production was attributed to the blockage of ROS-induced activation of mitogen-activated protein kinase (MAPK), ERK1/2 and p38 signaling pathways is involved in NETosis (Yang et al. 2019).

Beside neuropathy, NETosis is thought to be associated with the development of diabetes-induced retinopathy, since inflammation has been described as a key player in the onset of this microvascular complication. In fact, a case-control study investigated this hypothesis and results showed that NET formation markers, including circulating DNA-histone complex and polymorphonuclear neutrophil elastase levels, were elevated in patients with diabetic retinopathy as compared to diabetic patients without retinopathy. Interestingly, a multivariable logistic regression analysis, adjusted for fasting blood glucose and HbA1c levels, revealed that NET formation markers were significant independent risk factors of retinopathy (Park et al. 2016). These observations highlight the importance of NETs as a potential therapeutic target in diabetic retinopathy.

In parallel, NETs formation is considered to be associated with NADPH oxidases-induced ROS generation. In fact, neutrophils isolated from healthy individuals were exposed to increasing concentrations of glucose preloaded with $2^{\prime}, 7^{\prime}$-dichlorofluorescein diacetate (DCFH-DA) and dihydroethidium (DHE). Upon high glucose stimulation, neutrophils displayed increased ROS levels supporting the fact that NETs formation is associated with hyperglycemiainduced overproduction of ROS. Accordingly, pretreatment with either DPI or apocynin, both inhibitors of NADPH oxidase, reduced NETs formation and the release of extracellular DNA in neutrophils exposed to high glucose (Wang et al. 2018). Furthermore, NETs were present in the sera of patients with diabetic retinopathy irrespective of the disease stage (Wang et al. 2018). As observed in previous studies on wound healing, these findings suggest that NADPH oxidases are crucial in high glucose-induced NET production in diabetes-induced retinopathy.

Besides the role of NETosis in wound healing and retinopathy, increasing evidence highlights NETs formation as a risk factor for other microvascular complications. In fact, a recent study has shown that serum MPO-DNA complex level, a marker of NETs, was elevated in well-controlled patients with type 2 diabetes treated with several $(\geq 3)$ antidiabetic drugs as compared to patients treated with less $(<3)$ antidiabetic drugs and

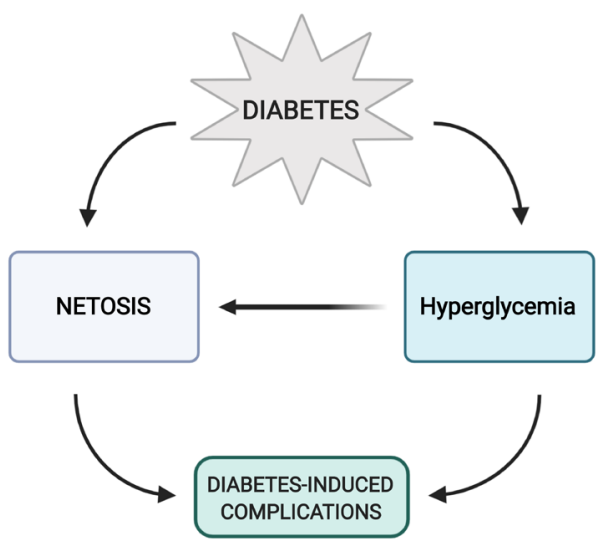

\section{Figure 2}

Proposed crosstalk between hyperglycemia and NETosis in diabetesinduced complications. A full-colour version of this figure is available at https://doi.org/10.1530/JME-20-0128.

healthy controls. More importantly, the serum MPODNA complex levels were positively correlated with clinical/laboratory parameters. which are known to be risk markers for microvascular complications including diabetic nephropathy or diabetic kidney disease including BMI and urinary albumin (Miyoshi et al. 2016). These findings suggest that NETs formation may serve as a risk marker for diabetes-induced renal complications in wellcontrolled patients. Despite these observations, further research is needed to better elucidate the role of NETs in diabetic nephropathy. However, based on data from our group and others, the role of NADPH oxidases in diabetesinduced microvascular complications, specifically diabetic nephropathy, has been well documented (Eid et al. 2009, 2010, 2013a,b, 2016, 2020). Since NETosis and NETs formation were shown to be highly dependent on NADPH-induced ROS production in neuropathy and retinopathy, one can advance the hypothesis that NETosis may be considered as a key player in the onset and development of diabetic kidney disease. However, it is important to mention that the role of NADPH oxidases is site and disease specific. For instance, it has been previously shown that NADPH oxidase 4 (NOX4) mediates protection against chronic load-induced stress in the heart and is essential for full development of eccentric cardiac hypertrophy and remodeling of the heart during chronic volume overload (Zhang et al. 2010, Schnelle et al. 2019). Furthermore, NOX4 has been shown to have an anti-atherosclerotic effect; hence, NOX4 inhibitors should be carefully assessed for cardiovascular side effects (Schürmann et al. 2015). 


\section{Conclusion}

This review highlights the important role that NETosis plays in the pathogenesis of diabetes and advances its role in diabetes-induced microvascular complications (Fig. 2). NETosis, or the process of NETs formation, has been shown to be activated by a wide array of proinflammatory cytokines and reactive oxygen species, notably in diabetes, which is characterized by a state of low-grade chronic inflammation. It has also been reported that a number of redox-sensitive pathways, including the NADPH oxidases pathways, orchestrate the key pathological events related to diabetes-induced microvascular complications, thus recognizing the role of NADPH oxidases-induced ROS as the possible bridge between NETosis and diabetes. Further studies aiming to better understand the exact mechanisms behind hyperglycemia-induced NETosis would be an essential step not only toward designating NETosis as a possible marker for the progression of diabetes, but also toward uncovering the likely therapeutic benefits of targeting NETosis in controlling the progression of diabetes-induced microvascular complications.

\section{Declaration of interest}

The authors declare that there is no conflict of interest that could be perceived as prejudicing the impartiality of this review.

\section{Funding}

$\mathrm{R} \mathrm{N}$ is funded by the American University of Beirut Faculty of Medicine Biomedical Sciences graduate program. ST A, H K K and A A E are supported by the American University of Beirut Medical Practice Plan regular research grant.

\section{Author contribution statement}

A A E and $\mathrm{H} \mathrm{K} \mathrm{K} \mathrm{conceived} \mathrm{and} \mathrm{designed} \mathrm{the} \mathrm{review} \mathrm{article} \mathrm{and} \mathrm{approved}$ the final version to be submitted. R N, W S A and A H F performed the literature review. R N and W S A analyzed and interpreted the data; wrote multiple subsections of the manuscript; and revised the manuscript for intellectual content. S T A reviewed and improved the entire manuscript.

\section{Acknowledgement}

Figures created with BioRender.com.

\section{References}

Alexiewicz JM, Kumar D, Smogorzewski M, Klin M \& Massry SG 1995 Polymorphonuclear leukocytes in non-insulin-dependent diabetes mellitus: abnormalities in metabolism and function. Annals of Internal Medicine 123 919-924. (https://doi.org/10.7326/0003-4819123-12-199512150-00004)
Behnen M, Leschczyk C, Moller S, Batel T, Klinger M, Solbach W \& Laskay T 2014 Immobilized immune complexes induce neutrophil extracellular trap release by human neutrophil granulocytes via FcgammaRIIIB and Mac-1. Journal of Immunology 193 1954-1965. (https://doi.org/10.4049/jimmunol.1400478)

Brinkmann V \& Zychlinsky A 2007 Beneficial suicide: why neutrophils die to make NETs. Nature Reviews: Microbiology 5 577-582. (https:// doi.org/10.1038/nrmicro1710)

Brinkmann V, Reichard U, Goosmann C, Fauler B, Uhlemann Y, Weiss DS, Weinrauch Y \& Zychlinsky A 2004 Neutrophi extracellular traps kill bacteria. Science 303 1532-1535. (https://doi. org/10.1126/science.1092385)

Byrd AS, O'Brien XM, Johnson CM, Lavigne LM \& Reichner JS 2013 An extracellular matrix-based mechanism of rapid neutrophil extracellular trap formation in response to Candida albicans. Journal of Immunology 190 4136-4148. (https://doi.org/10.4049/ jimmunol.1202671)

Callaghan BC, Price RS \& Feldman EL 2015 Distal symmetric polyneuropathy: a review. JAMA 314 2172-2181. (https://doi. org/10.1001/jama.2015.13611)

Carestia A, Frechtel G, Cerrone G, Linari MA, Gonzalez CD, Casais P \& Schattner M 2016 NETosis before and after hyperglycemic control in type 2 diabetes mellitus patients. PLOS ONE 11 e0168647. (https:// doi.org/10.1371/journal.pone.0168647)

Clark SR, Ma AC, Tavener SA, McDonald B, Goodarzi Z, Kelly MM, Patel KD, Chakrabarti S, McAvoy E, Sinclair GD, et al. 2007 Platelet TLR4 activates neutrophil extracellular traps to ensnare bacteria in septic blood. Nature Medicine 13 463-469. (https://doi.org/10.1038/ $\mathrm{nm} 1565)$

Cooper ME, Vranes D, Youssef S, Stacker SA, Cox AJ, Rizkalla B, Casley DJ, Bach LA, Kelly DJ \& Gilbert RE 1999 Increased renal expression of vascular endothelial growth factor (VEGF) and its receptor VEGFR-2 in experimental diabetes. Diabetes 48 2229-2239. (https://doi.org/10.2337/diabetes.48.11.2229)

Corsiero E, Pratesi F, Prediletto E, Bombardieri M \& Migliorini P 2016 NETosis as source of autoantigens in rheumatoid arthritis. Frontiers in Immunology 7 485. (https://doi.org/10.3389/fimmu.2016.00485)

Das SK, Yuan YF \& Li MQ 2018 Specific PKC betaII inhibitor: one stone two birds in the treatment of diabetic foot ulcers. Bioscience Reports 38 BSR20171459. (https://doi.org/10.1042/BSR20171459)

Delgado-Rizo V, Martinez-Guzman MA, Iniguez-Gutierrez L, GarciaOrozco A, Alvarado-Navarro A \& Fafutis-Morris M 2017 Neutrophil extracellular traps and its implications in inflammation: an overview. Frontiers in Immunology 8 81. (https://doi.org/10.3389/ fimmu.2017.00081)

Diana J, Simoni Y, Furio L, Beaudoin L, Agerberth B, Barrat F \& Lehuen A 2013 Crosstalk between neutrophils, B-1a cells and plasmacytoid dendritic cells initiates autoimmune diabetes. Nature Medicine 19 65-73. (https://doi.org/10.1038/nm.3042)

Eid AA, Gorin Y, Fagg BM, Maalouf R, Barnes JL, Block K \& Abboud HE 2009 Mechanisms of podocyte injury in diabetes: role of cytochrome P450 and NADPH oxidases. Diabetes 58 1201-1211. (https://doi. org/10.2337/db08-1536)

Eid AA, Ford BM, Block K, Kasinath BS, Gorin Y, Ghosh-Choudhury G, Barnes JL \& Abboud HE 2010 AMP-activated protein kinase (AMPK) negatively regulates Nox4-dependent activation of p53 and epithelial cell apoptosis in diabetes. Journal of Biological Chemistry $\mathbf{2 8 5}$ 37503-37512. (https://doi.org/10.1074/jbc.M110.136796)

Eid AA, Lee DY, Roman LJ, Khazim K \& Gorin Y 2013a Sestrin 2 and AMPK connect hyperglycemia to Nox4-dependent endothelial nitric oxide synthase uncoupling and matrix protein expression. Molecular and Cellular Biology 33 3439-3460. (https://doi.org/10.1128/ MCB.00217-13)

Eid AA, Ford BM, Bhandary B, de Cassia Cavaglieri R, Block K, Barnes JL, Gorin Y, Choudhury GG \& Abboud HE 2013 $b$ Mammalian target of rapamycin regulates Nox4-mediated podocyte depletion in diabetic 
renal injury. Diabetes 62 2935-2947. (https://doi.org/10.2337/db121504)

Eid S, Boutary S, Braych K, Sabra R, Massaad C, Hamdy A, Rashid A, Moodad S, Block K, Gorin Y, et al. 2016 mTORC2 signaling regulates Nox4-induced podocyte depletion in diabetes. Antioxidants and Redox Signaling 25 703-719. (https://doi.org/10.1089/ ars.2015.6562)

Eid SA, El Massry M, Hichor M, Haddad M, Grenier J, Dia B, Barakat R, Boutary S, Chanal J, Aractingi S, et al. 2020 Targeting the NADPH oxidase-4 and liver $\mathrm{X}$ receptor pathway preserves Schwann cell integrity in diabetic mice. Diabetes 69 448-464. (https://doi. org/10.2337/db19-0517)

Fadini GP, Menegazzo L, Rigato M, Scattolini V, Poncina N, Bruttocao A, Ciciliot S, Mammano F, Ciubotaru CD, Brocco E, et al. 2016 NETosis delays diabetic wound healing in mice and humans. Diabetes $\mathbf{6 5}$ 1061-1071. (https://doi.org/10.2337/db15-0863)

Farrera C \& Fadeel B 2013 Macrophage clearance of neutrophil extracellular traps is a silent process. Journal of Immunology 191 2647-2656. (https://doi.org/10.4049/jimmunol.1300436)

Fontayne A, Dang PM, Gougerot-Pocidalo MA \& El-Benna J 2002 Phosphorylation of p47phox sites by PKC alpha, beta II, delta, and zeta: effect on binding to p22phox and on NADPH oxidase activation. Biochemistry 41 7743-7750. (https://doi.org/10.1021/ bi011953s)

Forbes JM \& Cooper ME 2013 Mechanisms of diabetic complications. Physiological Reviews 93 137-188. (https://doi.org/10.1152/ physrev.00045.2011)

Garcia-Romo GS, Caielli S, Vega B, Connolly J, Allantaz F, Xu Z, Punaro M, Baisch J, Guiducci C, Coffman RL, et al. 2011 Netting neutrophils are major inducers of type I IFN production in pediatric systemic lupus erythematosus. Science Translational Medicine $\mathbf{3}$ 73ra20. (https://doi.org/10.1126/scitranslmed.3001201)

Hakkim A, Furnrohr BG, Amann K, Laube B, Abed UA, Brinkmann V, Herrmann M, Voll RE \& Zychlinsky A 2010 Impairment of neutrophil extracellular trap degradation is associated with lupus nephritis. PNAS 107 9813-9818. (https://doi.org/10.1073/ pnas.0909927107)

Hamam HJ \& Palaniyar N 2019a Histone deacetylase inhibitors dosedependently switch neutrophil death from NETosis to apoptosis. Biomolecules 9 184. (https://doi.org/10.3390/biom9050184)

Hamam HJ \& Palaniyar N 2019b Post-translational modifications in NETosis and NETs-mediated diseases. Biomolecules 9 369. (https://doi. org/10.3390/biom9080369)

Hamam HJ, Khan MA \& Palaniyar N 2019 Histone acetylation promotes neutrophil extracellular trap formation. Biomolecules 9 32. (https:// doi.org/10.3390/biom9010032)

Harsunen MH, Puff R, D'Orlando O, Giannopoulou E, Lachmann L, Beyerlein A, von Meyer A \& Ziegler AG 2013 Reduced blood leukocyte and neutrophil numbers in the pathogenesis of type 1 diabetes. Hormone and Metabolic Research 45 467-470. (https://doi. org/10.1055/s-0032-1331226)

Joshi MB, Lad A, Bharath Prasad AS, Balakrishnan A, Ramachandra L \& Satyamoorthy K 2013 High glucose modulates IL-6 mediated immune homeostasis through impeding neutrophil extracellular trap formation. FEBS Letters $\mathbf{5 8 7}$ 2241-2246. (https://doi.org/10.1016/j. febslet.2013.05.053)

Keshari RS, Jyoti A, Dubey M, Kothari N, Kohli M, Bogra J, Barthwal MK \& Dikshit M 2012 Cytokines induced neutrophil extracellular traps formation: implication for the inflammatory disease condition. PLoS ONE 7 e48111. (https://doi.org/10.1371/journal.pone.0048111)

King P, Peacock I \& Donnelly R 1999 The UK prospective diabetes study (UKPDS): clinical and therapeutic implications for type 2 diabetes. British Journal of Clinical Pharmacology 48 643-648. (https://doi. org/10.1046/j.1365-2125.1999.00092.x)

Lewis HD, Liddle J, Coote JE, Atkinson SJ, Barker MD, Bax BD, Bicker KL, Bingham RP, Campbell M, Chen YH, et al. 2015
Inhibition of PAD4 activity is sufficient to disrupt mouse and human NET formation. Nature Chemical Biology 11 189-191. (https://doi. org/10.1038/nchembio.1735)

Li P, Li M, Lindberg MR, Kennett MJ, Xiong N \& Wang Y 2010 PAD4 is essential for antibacterial innate immunity mediated by neutrophil extracellular traps. Journal of Experimental Medicine 207 1853-1862. (https://doi.org/10.1084/jem.20100239)

Mantovani A, Cassatella MA, Costantini C \& Jaillon S 2011 Neutrophils in the activation and regulation of innate and adaptive immunity. Nature Reviews: Immunology 11 519-531. (https://doi.org/10.1038/ nri3024)

Menegazzo L, Ciciliot S, Poncina N, Mazzucato M, Persano M, Bonora B, Albiero M, Vigili de Kreutzenberg S, Avogaro A \& Fadini GP 2015 NETosis is induced by high glucose and associated with type 2 diabetes. Acta Diabetologica 52 497-503. (https://doi.org/10.1007/ s00592-014-0676-x)

Menegazzo L, Scattolini V, Cappellari R, Bonora BM, Albiero M, Bortolozzi M, Romanato F, Ceolotto G, Vigili de Kreutzeberg S, Avogaro A, et al. 2018 The antidiabetic drug metformin blunts NETosis in vitro and reduces circulating NETosis biomarkers in vivo. Acta Diabetologica 55 593-601. (https://doi.org/10.1007/s00592-0181129-8)

Miyoshi A, Yamada M, Shida H, Nakazawa D, Kusunoki Y, Nakamura A, Miyoshi H, Tomaru U, Atsumi T \& Ishizu A 2016 Circulating neutrophil extracellular trap levels in well-controlled Type 2 diabetes and pathway involved in their formation induced by high-dose glucose. Pathobiology 83 243-251. (https://doi. org $/ 10.1159 / 000444881)$

Nathan DM \& DCCT/EDIC Research Group 2014 The diabetes control and complications trial/epidemiology of diabetes interventions and complications study at 30 years: overview. Diabetes Care 37 9-16. (https://doi.org/10.2337/dc13-2112)

Neeli I, Khan SN \& Radic M 2008 Histone deimination as a response to inflammatory stimuli in neutrophils. Journal of Immunology $\mathbf{1 8 0}$ 1895-1902. (https://doi.org/10.4049/jimmunol.180.3.1895)

Neeli I, Dwivedi N, Khan S \& Radic M 2009 Regulation of extracellular chromatin release from neutrophils. Journal of Innate Immunity $\mathbf{1}$ 194-201. (https://doi.org/10.1159/000206974)

Papayannopoulos V 2018 Neutrophil extracellular traps in immunity and disease. Nature Reviews: Immunology 18 134-147. (https://doi. org/10.1038/nri.2017.105)

Papayannopoulos V, Metzler KD, Hakkim A \& Zychlinsky A 2010 Neutrophil elastase and myeloperoxidase regulate the formation of neutrophil extracellular traps. Journal of Cell Biology 191 677-691. (https://doi.org/10.1083/jcb.201006052)

Park JH, Kim JE, Gu JY, Yoo HJ, Park SH, Kim YI, Nam-Goong IS, Kim ES \& Kim HK 2016 Evaluation of circulating markers of neutrophil extracellular trap (NET) formation as risk factors for diabetic retinopathy in a Case-Control Association Study. Experimental and Clinical Endocrinology and Diabetes 124 557-561. (https://doi. org/10.1055/s-0042-101792)

Pilsczek FH, Salina D, Poon KK, Fahey C, Yipp BG, Sibley CD, Robbins SM, Green FH, Surette MG, Sugai M, et al. 2010 A novel mechanism of rapid nuclear neutrophil extracellular trap formation in response to Staphylococcus aureus. Journal of Immunology $\mathbf{1 8 5}$ 7413-7425. (https://doi.org/10.4049/jimmunol.1000675)

Qin J, Fu S, Speake C, Greenbaum CJ \& Odegard JM 2016 NETosisassociated serum biomarkers are reduced in type 1 diabetes in association with neutrophil count. Clinical and Experimental Immunology 184 318-322. (https://doi.org/10.1111/cei.12783)

Rodriguez-Espinosa O, Rojas-Espinosa O, Moreno-Altamirano MM, Lopez-Villegas EO \& Sanchez-Garcia FJ 2015 Metabolic requirements for neutrophil extracellular traps formation. Immunology $\mathbf{1 4 5}$ 213-224. (https://doi.org/10.1111/imm.12437)

Rosales C, Lowell CA, Schnoor M \& Uribe-Querol E 2017 Neutrophils: their role in innate and adaptive immunity 2017. Journal of 
Immunology Research 2017 9748345. (https://doi. org/10.1155/2017/9748345)

Schnelle M, Sawyer I, Anilkumar N, Mohamed BA, Richards DA, Toischer K, Zhang M, Catibog N, Sawyer G, Mongue-Din H, et al. 2019 NADPH oxidase-4 promotes eccentric cardiac hypertrophy in response to volume overload. Cardiovascular Research [epub]. (https:// doi.org/10.1093/cvr/cvz331)

Schorn C, Janko C, Latzko M, Chaurio R, Schett G \& Herrmann M 2012 Monosodium urate crystals induce extracellular DNA traps in neutrophils, eosinophils, and basophils but not in mononuclear cells. Frontiers in Immunology 3 277. (https://doi.org/10.3389/ fimmu.2012.00277)

Schürmann C, Rezende F, Kruse C, Yasar Y, Löwe O, Fork C, van de Sluis B, Bremer R, Weissmann N, Shah AM, et al. 2015 The NADPH oxidase Nox4 has anti-atherosclerotic functions. European Heart Journal 36 3447-3456. (https://doi.org/10.1093/eurheartj/ehv460)

Semeraro F, Ammollo CT, Morrissey JH, Dale GL, Friese P, Esmon NL \& Esmon CT 2011 Extracellular histones promote thrombin generation through platelet-dependent mechanisms: involvement of platelet TLR2 and TLR4. Blood 118 1952-1961. (https://doi.org/10.1182/ blood-2011-03-343061)

Skyler JS 2004 Effects of glycemic control on diabetes complications and on the prevention of diabetes. Clinical Diabetes 22 162-166. (https:// doi.org/10.2337/diaclin.22.4.162)

Takei H, Araki A, Watanabe H, Ichinose A \& Sendo F 1996 Rapid killing of human neutrophils by the potent activator phorbol 12-myristate 13-acetate (PMA) accompanied by changes different from typical apoptosis or necrosis. Journal of Leukocyte Biology 59 229-240. (https://doi.org/10.1002/jlb.59.2.229)

Terry T, Raravikar K, Chokrungvaranon N \& Reaven PD 2012 Does aggressive glycemic control benefit macrovascular and microvascular disease in Type 2 diabetes? Insights from Accord, ADVANCE, and VADT. Current Cardiology Reports 14 79-88. (https://doi.org/10.1007/ s11886-011-0238-6)

Valle A, Giamporcaro GM, Scavini M, Stabilini A, Grogan P, Bianconi E, Sebastiani G, Masini M, Maugeri N, Porretti L, et al. 2013 Reduction of circulating neutrophils precedes and accompanies type 1 diabetes. Diabetes 62 2072-2077. (https://doi.org/10.2337/db12-1345)

Volmer-Thole M \& Lobmann R 2016 Neuropathy and diabetic foot syndrome. International Journal of Molecular Sciences 17. (https://doi. org/10.3390/ijms17060917)
Wang Y, Xiao Y, Zhong L, Ye D, Zhang J, Tu Y, Bornstein SR, Zhou Z, Lam KS \& Xu A 2014 Increased neutrophil elastase and proteinase 3 and augmented NETosis are closely associated with beta-cell autoimmunity in patients with type 1 diabetes. Diabetes $\mathbf{6 3}$ 4239-4248. (https://doi.org/10.2337/db14-0480)

Wang L, Zhou X, Yin Y, Mai Y, Wang D \& Zhang X 2018 Hyperglycemia induces neutrophil extracellular traps formation through an NADPH oxidase-dependent pathway in diabetic retinopathy. Frontiers in Immunology 9 3076. (https://doi. org/10.3389/fimmu.2018.03076)

Wong SL, Demers M, Martinod K, Gallant M, Wang Y, Goldfine AB, Kahn CR \& Wagner DD 2015 Diabetes primes neutrophils to undergo NETosis, which impairs wound healing. Nature Medicine 21 815-819. (https://doi.org/10.1038/nm.3887)

Yalavarthi S, Gould TJ, Rao AN, Mazza LF, Morris AE, Nunez-Alvarez C, Hernandez-Ramirez D, Bockenstedt PL, Liaw PC, Cabral AR, et al. 2015 Release of neutrophil extracellular traps by neutrophils stimulated with antiphospholipid antibodies: a newly identified mechanism of thrombosis in the antiphospholipid syndrome. Arthritis and Rheumatology 67 2990-3003. (https://doi.org/10.1002/ art.39247)

Yang H, Biermann MH, Brauner JM, Liu Y, Zhao Y \& Herrmann M 2016 New insights into neutrophil extracellular traps: mechanisms of formation and role in inflammation. Frontiers in Immunology $\mathbf{7} 302$. (https://doi.org/10.3389/fimmu.2016.00302)

Yang CT, Chen L, Chen WL, Li N, Chen MJ, Li X, Zheng X, Zhao YZ, Wu YX, Xian M, et al. 2019 Hydrogen sulfide primes diabetic wound to close through inhibition of NETosis. Molecular and Cellular Endocrinology 480 74-82. (https://doi.org/10.1016/j. mce.2018.10.013)

Yipp BG \& Kubes P 2013 NETosis: how vital is it? Blood 122 2784-2794. (https://doi.org/10.1182/blood-2013-04-457671)

Yousefi S, Mihalache C, Kozlowski E, Schmid I \& Simon HU 2009 Viable neutrophils release mitochondrial DNA to form neutrophil extracellular traps. Cell Death and Differentiation 16 1438-1444. (https://doi.org/10.1038/cdd.2009.96)

Zhang M, Brewer AC, Schröder K, Santos CXC, Grieve DJ, Wang M, Anilkumar N, Yu B, Dong X, Walker SJ, et al. 2010 NADPH oxidase-4 mediates protection against chronic load-induced stress in mouse hearts by enhancing angiogenesis. PNAS 107 18121-18126. (https:// doi.org/10.1073/pnas.1009700107)

Received in final form 30 July 2020

Accepted 3 September 2020

Accepted Manuscript published online 4 September 2020 (c) 2020 Society for Endocrinology Published by Bioscientifica Ltd. Printed in Great Britain 